\title{
Surgery After Response to Chemotherapy for Locally Advanced Pancreatic Ductal Adenocarcinoma: A Guide for Management
}

Zhi Ven Fong, MD, MPH, and Cristina R. Ferrone, MD

\begin{abstract}
Because of the biologic aggressiveness and late presentation of pancreatic ductal adenocarcinoma (PDAC), up to $80 \%$ of patients have locally advanced or metastatic disease at presentation. The success of multiagent chemotherapy regimens in the management of metastatic disease has been translated to patients with locally advanced PDAC. Both FOLFIRINOX (fluorouracil/folinic acid/irinotecan/oxaliplatin) and gemcitabine/nab-paclitaxel are used to downstage locally advanced PDAC to render it eligible for resection with curative intent. This paradigm shift has significantly expanded the pool of patients who are eligible for resection with curative intent. However, the generalizability of present studies and the patient selection process are unclear. This article provides an evidence-based review of patient selection considerations and management algorithms, and details our institution's approach to patients with locally advanced PDAC after preoperative chemotherapy.
\end{abstract}

J Natl Compr Canc Netw 2021;19(4):459-467 doi: 10.6004/jnccn.2021.7009

Department of Surgery, Massachusetts General Hospital, Harvard Medical School, Boston, Massachusetts
The incidence of pancreatic adenocarcinoma (PDAC) continues to increase, with an estimated 57,000 newly diagnosed patients in the United States in 2021. ${ }^{1}$ It is currently the fourth leading cause of cancer death and is projected to be the second leading cause by $2030 .^{2}$ PDAC is biologically aggressive and often presents at a later stage, with up to $80 \%$ of patients found to have locally advanced cancer or distant metastases at the time of initial evaluation. $^{3}$

In 2011 and 2013, the treatment horizon for patients with metastatic PDAC significantly changed with the multiagent chemotherapy regimens fluorouracil/folinic acid/irinotecan/oxaliplatin (FOLFIRINOX) ${ }^{4}$ and gemcitabine/nab-paclitaxel,${ }^{5}$ respectively. Median survival for patients with metastatic PDAC increased from 6.8 to 11.1 months with FOLFIRINOX ${ }^{4}$ and from 6.7 to 8.5 months with gemcitabine/nab-paclitaxel. ${ }^{5}$ With a historical median survival of 11 to 12 months for patients with locally advanced PDAC, ${ }^{6}$ many physicians presumed that these patients had micrometastatic disease and would similarly benefit from these regimens. Several retrospective studies have been published showing the effectiveness of total neoadjuvant therapy (TNT) using FOLFIRINOX followed by chemoradiotherapy and surgical resection., Institutional series have even shown superior survival in patients with locally advanced disease undergoing TNT followed by resection when compared with patients with resectable disease undergoing resection. ${ }^{9}$ TNT allows for improved delivery rates of effective chemotherapy and radiotherapy, an increased ability to achieve an R0 resection, and selection of patients with locally advanced PDAC with favorable tumor biology. 9,10

Although retrospective series can provide data to support the rational design of clinical trials, these data have significant limitations. Phase II clinical trials, although not as robust as phase III trials, do provide more robust data than retrospective series. In a single-institution phase II trial, 49 patients with locally advanced PDAC were given 8 cycles of FOLFIRINOX followed by 50.4 Gy of photon radiation with capecitabine and losartan. ${ }^{11}$ Of these patients, 39 were brought to the operating room and 34 (69\%) had their cancer removed. Of these 34 patients, 30 (88\%) had 
an R0 resection and only 9 (26\%) had N1 disease. In patients who underwent resection, median progression-free survival (PFS) and overall survival (OS) were 21.3 and 33 months, respectively. These outcomes are significantly improved compared with the historical OS of 11 to 12 months. Neoadjuvant therapy has since been shown to be associated with a downstaging-to-resection rate of $>30 \%$ in selected patients with locally advanced tumors, and with survival comparable to or better than that of patients with initially resectable disease. ${ }^{7,8}$

\section{Definition of Locally Advanced PDAC}

The historic definition of locally advanced PDAC has been inconsistent, mainly because of the considerable debate and controversy about which patients have truly resectable or unresectable disease, which is dependent on physicians' interpretation of cross-sectional imaging when viewing the mass's relation to major visceral vessels, the individual surgeon's skills, and institutional experiences. In an effort to standardize its definition, 4 organizational bodies have proposed classification systems: NCCN, the Americas Hepato-Pancreato-Biliary Association/Society of Surgical Oncology/Society for Surgery of the Alimentary Tract, The University of Texas MD Anderson Cancer Center, and the Alliance for Clinical Trials in Oncology (Alliance), as depicted in Table 1. Even so, there has been ambiguity about what constitutes abutment and encasement, with the Alliance group eliminating the subjectivity surrounding these terms by defining them down to the degree of the tumor-vessel interface. For the purpose of clinical trials and research, these definitions are important for a clear understanding of the study population and for comparing outcomes across different trials. For the purpose of clinical management, these definitions

\section{Table 1: Differences in the Definition of Borderline and Locally Advanced PDAC}

\begin{tabular}{|c|c|c|c|c|}
\hline & $\mathrm{NCCN}^{25}$ & AHPBA/SSO/SSAT $T^{57}$ & MDACC Classification ${ }^{38}$ & Alliance $^{58}$ \\
\hline \multicolumn{5}{|l|}{ Borderline } \\
\hline Arterial & $\begin{array}{l}\text { Head/Uncinate process: } \\
\text { Solid tumor contact with } \\
\text { CHA without extension to } \\
\text { CA or HA bifurcation } \\
\text { Contact with the SMA } \\
\leq 180^{\circ} \\
\text { Contact with variant } \\
\text { arterial anatomy } \\
\text { Pancreatic body/tail: } \\
\text { Solid tumor contact with } \\
\text { CA } \leq 180^{\circ} \\
\text { Contact with CA }>180^{\circ} \\
\text { without involvement of } \\
\text { aorta and with intact and } \\
\text { uninvolved GDA }\end{array}$ & $\begin{array}{l}\text { Abutment of SMA } \\
\text { Abutment or short- } \\
\text { segment encasement } \\
\text { of CHA } \\
\text { No abutment or } \\
\text { encasement of CA }\end{array}$ & $\begin{array}{l}\text { Abutment of SMA } \\
\text { Abutment of CHA } \\
\text { Abutment of CA }\end{array}$ & $\begin{array}{l}\text { Solid tumor contact } \\
\text { with SMA }<180^{\circ} \\
\text { Reconstructable, } \\
\text { solid tumor contact } \\
\text { of CHA of any degree } \\
\text { Solid tumor contact } \\
\text { with CA }<180^{\circ}\end{array}$ \\
\hline Venous & $\begin{array}{l}\text { Solid tumor contact with } \\
\text { SMV/PV }>180^{\circ} \\
\text { Contact of } \leq 180^{\circ} \text { with } \\
\text { contour irregularity of vein } \\
\text { or thrombosis of vein but } \\
\text { with suitable vessel } \\
\text { proximal and distal to site } \\
\text { of involvement } \\
\text { Contact with IVC }\end{array}$ & $\begin{array}{l}\text { Abutment, encasement, or } \\
\text { occlusion of SMV/PV }\end{array}$ & $\begin{array}{l}\text { Short-segment occlusion } \\
\text { of SMV/PV }\end{array}$ & $\begin{array}{l}\text { Solid tumor contact } \\
\text { with } \mathrm{SMV} / \mathrm{PV}>180^{\circ} \\
\text { or reconstructable } \\
\text { occlusion }\end{array}$ \\
\hline \multicolumn{5}{|c|}{ Locally advanced } \\
\hline Arterial & $\begin{array}{l}\text { Head/Uncinate process: } \\
\text { Solid tumor contact with } \\
\text { SMA }>180^{\circ} \\
\text { Contact with CA }>180^{\circ} \\
\text { Pancreatic body/tail: } \\
\text { Solid tumor contact with } \\
\text { SMA/CA }>180^{\circ} \\
\text { Contact with CA and } \\
\text { aortic involvement }\end{array}$ & $\begin{array}{l}\text { Encasement of SMA } \\
\text { Long-segment encasement } \\
\text { of CHA }\end{array}$ & $\begin{array}{l}\text { Encasement of SMA } \\
\text { Long-segment encasement } \\
\text { of CHA }\end{array}$ & $\begin{array}{l}\text { Solid tumor contact } \\
\text { with SMA } \geq 180^{\circ}\end{array}$ \\
\hline Venous & $\begin{array}{l}\text { Unreconstructible } \\
\text { SMV/PV due to tumor } \\
\text { involvement or occlusion }\end{array}$ & $\begin{array}{l}\text { Unreconstructable } \\
\text { SMV/PV due to tumor } \\
\text { involvement or occlusion }\end{array}$ & $\begin{array}{l}\text { Unreconstructable } \\
\text { SMV/PV due to tumor } \\
\text { involvement or occlusion }\end{array}$ & $\begin{array}{l}\text { Unreconstructable } \\
\text { SMV/PV due to } \\
\text { tumor involvement } \\
\text { or occlusion }\end{array}$ \\
\hline
\end{tabular}

Abbreviations: AHPBA, Americas Hepato-Pancreato-Biliary Association; Alliance, Alliance for Clinical Trials in Oncology; CA, celiac axis; $\mathrm{CHA}$, common hepatic artery; GDA, gastroduodenal artery; HA, hepatic artery; MDACC, The University of Texas MD Anderson Cancer Center; PV, portal vein; SMA, superior mesenteric artery; SMV, superior mesenteric vein; SSAT, Society for Surgery of the Alimentary Tract; SSO, Society of Surgical Oncology. 
are less helpful, because imaging after neoadjuvant therapy is less reliable, which is discussed in the following sections.

Given this change in effective systemic therapy for patients with locally advanced PDAC, the pool of patients eligible for resection with curative intent has greatly expanded. However, the generalizability of present studies and the patient selection process are unclear. How do we gauge tumor response to preoperative chemotherapy? Which patients should be selected to undergo operative exploration? What are the treatment options in the operating room? This review presents an evidence-based guide for the management of patients with locally advanced PDAC who have undergone preoperative chemotherapy, aiming to guide clinicians to better understand and refine patient selection to bolster these promising results.

\section{Patient Selection for Surgical Resection}

Given the complexity of managing patients with locally advanced PDAC, patients should be presented at a multidisciplinary tumor board and jointly managed by a team of medical, surgical, and radiation oncologists. ${ }^{12}$

Ferrone et $\mathrm{al}^{9}$ at Massachusetts General Hospital described the first series in the literature documenting the failure of standard imaging to determine resectability after neoadjuvant FOLFIRINOX and chemoradiotherapy in patients who presented with borderline or locally advanced PDAC. In the absence of metastatic progression, only a small proportion of patients who have received TNT will show a radiographic response, with most showing stable radiographic disease. In a blinded review of CT scans, $92 \%$ of patients with locally advanced PDAC who were deemed radiographically unresectable after neoadjuvant therapy had an R0 resection (Figure 1). ${ }^{9}$ This finding was hypothesized to be secondary to the inability of cross-sectional imaging to distinguish posttreatment fibrosis from viable cancer. Multiple other studies have since corroborated the lack of correlation between cross-sectional imaging and resectability rates, pathologic response, or OS. ${ }^{13-17}$

To improve the ability of imaging to determine response, FDG PET/CT, FDG PET/MRI, and radiomics are being explored by multiple groups. A recent study from the Medical College of Wisconsin indicated that patients with PDAC who had high FDG uptake on FDG PET/CT at diagnosis but normalized uptake preoperatively had significantly improved OS compared with patients with persistently elevated FDG uptake. ${ }^{18}$

Similarly, FDG PET/MRI is being explored as a method of measuring response to neoadjuvant therapy. ${ }^{19} \mathrm{~A}$ group at the University of California San Francisco compared PET/MRI at diagnosis and at 4 weeks postinitiation of treatment, with a decrease in uptake correlating with a significantly improved PFS and OS. ${ }^{20}$ This early marker of response is important so that nonresponders can change treatment regimens. Although PET scans have shown utility, there may be a larger potential in radiomics and machine learning algorithms. Algorithms to determine biologic subtypes and treatment responses are being actively developed. ${ }^{21,22}$

Conversely, CA 19-9 levels have emerged as a reliable predictor of resectability after preoperative chemotherapy. One study reported that a $>50 \%$ response in CA 19-9 level was independently associated with improved survival (hazard ratio $[\mathrm{HR}], 0.26 ; P<.0001) .{ }^{23}$ Other researchers have reported that a normal posttreatment CA 19-9 level, irrespective of whether the pretreatment CA 19-9 level was normal or elevated, predicted recurrence-free survival (HR, 0.41; $P<.001)$ and OS (HR, 0.49; $P<.01){ }^{14}$ Similarly, another study from the Medical College of Wisconsin analyzed pretreatment CA 19-9 levels, the magnitude of posttreatment change in CA 19-9 levels, and normalization in CA 19-9 levels, showing that the latter is the single most important prognostic marker of long-term survival after surgical resection. ${ }^{24}$ Accordingly, the NCCN Clinical Practice Guidelines in Oncology
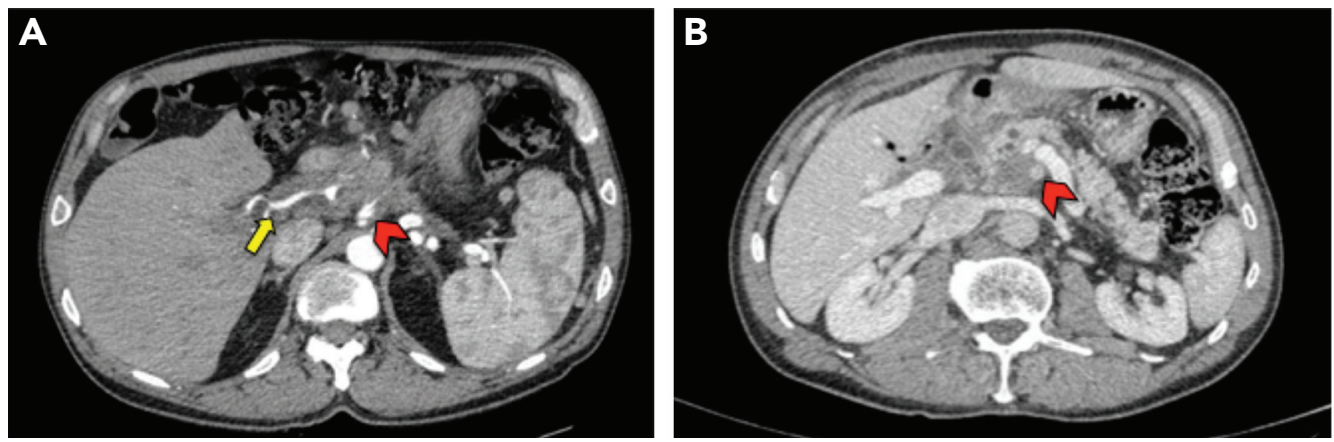

Figure 1. (A) Posttreatment imaging of a locally advanced pancreatic body mass circumferentially encasing the left hepatic artery (yellow arrow) and celiac axis (red arrowhead). The patient's CA 19-9 level was $129 \mathrm{U} / \mathrm{L}$ and decreased to $29 \mathrm{U} / \mathrm{L}$ posttreatment, prompting surgical exploration 
(NCCN Guidelines) for Pancreatic Adenocarcinoma ${ }^{25}$ state that a $>50 \%$ decrease in CA 19-9 levels and clinical improvement in performance status and symptoms would indicate response to therapy and warrant surgical exploration. It is also worth noting that approximately $5 \%$ to $10 \%$ of patients with pancreatic cancer do not express CA 19-9 because they do not have the Lewis antigen, despite having a high tumor burden. ${ }^{26,27}$ Therefore, we recommend proceeding with surgical exploration in patients with downtrending or stable CA 19-9 levels in the absence of radiographic progression of the tumor or metastatic disease.

Several novel methods have been reported to determine response to neoadjuvant therapy and subsequently candidacy for surgical resection. Circulating tumor DNA (ctDNA) is emerging as a promising biomarker for diagnosis, prognosis, and treatment response in multiple types of tumors. Specifically in patients with borderline and locally advanced PDAC receiving neoadjuvant FOLFIRINOX and radiotherapy, the rate of R0/LN-negative resections was significantly higher in patients with an undetectable preoperative ctDNA level compared with those with a detectable preoperative ctDNA level (R0/ LN-negative rate, $80 \%$ vs $38 \%$, respectively; $P=.009$ ). ${ }^{28}$ Similarly, a Norwegian pilot study using KRAS mutations in plasma as a surrogate for ctDNA showed that patients who were KRAS mutation-positive before chemotherapy exhibited a significantly higher rate of disease progression during follow-up compared with patients who were KRAS mutation-negative (90\% vs $25 \% ; P=.01) .{ }^{29}$ Furthermore, changes in KRAS mutation levels corresponded both with radiologic follow-up data and CA 19-9 levels. The potential of ctDNA is also reflected in the finding that in the metastatic setting, ctDNA levels have increased before progression becomes visible on imaging. ${ }^{29,30}$ It is worth noting that ctDNA could be used to determine the presence of mutations, some of which may be found in future studies to be predictive of response to specific treatment regimens. ${ }^{29}$

As many of these modalities are further developed, their utility and practicality will become apparent. Currently, the decision to perform surgical exploration continues to be based on the lack of progression on imaging, patient performance status, and decreasing/normalization of CA 19-9 level.

\section{Surgical Resection Strategies and Considerations}

Intraoperative Staging: Clinical Utility of

Staging Laparoscopy

When the decision has been made to perform surgically exploration on selected patients with locally advanced PDAC after neoadjuvant therapy, a staging laparoscopy should be first performed to assess for any radiographically occult metastatic disease. Although improvements in cross-sectional imaging quality and systemic therapy have decreased the historic yield of staging laparoscopy, we found that this procedure still prevented unnecessary laparotomy in $24 \%$ of patients, especially if CA 19-9 levels were $>394 \mathrm{U} / \mathrm{L}$ (odds ratio, $3.1 ; P<.001$ ) and the cancer was in the body/tail of the pancreas (odds ratio, 1.8; $P=.06){ }^{31,32}$ In addition, patients with unsuspected metastases who were spared a laparotomy and only had a staging laparoscopy started palliative chemotherapy more quickly (17.9 vs 39.9 days; $P<.001$ ) and had an improved OS (11.4 vs 8.3 months; adjusted HR, 0.53; $P=.005)$ with no added lifetime incidence of cholangitis or gastric outlet obstruction. ${ }^{32}$ Notably, Peng et $\mathrm{al}^{33}$ assessed the yield of staging laparoscopy before initiation of neoadjuvant treatment and similarly reported a $25 \%$ rate of occult metastatic disease, which highlights the importance of staging laparoscopy being performed earlier in the treatment course as a separate procedure. If staging laparoscopy does not reveal any unexpected radiographically occult metastases, it is still important to inspect areas that are poorly visualized by laparoscopy, such as the dome of the liver and the lesser sac. Currently, no data support a meaningful survival advantage to resecting the primary PDAC in the setting of metastatic disease.

\section{Frozen Section Analysis}

Patients with PDAC who undergo an R0 resection have an improved OS, whether they are treatment-naïve or have received neoadjuvant therapy. Therefore, we recommend the liberal use of frozen section, especially in the areas of vascular involvement at the time of presentation. This recommendation is supported by the retrospective, dual-institutional analysis of 986 patients in which reresection to achieve negative margins based on frozen section analysis was associated with improved OS without an increase in morbidity or mortality. ${ }^{34}$ This result is particularly important in patients who have undergone TNT given that, as previously mentioned, cross-sectional imaging cannot differentiate posttherapy fibrosis from residual viable tumor, and that in the setting of positive margins there are treatment strategies that impact survival, which are discussed in this review. For these reasons, we recommend the liberal use of frozen section analysis of margins along the tumor-vessel interface.

\section{Resection}

In the setting of vascular involvement, where there may be macroscopically or microscopically positive margins, 3 main management strategies can be considered: (1) divestment or resection and reconstruction of the vessel involved, (2) intraoperative radiation (IORT) for 
either venous or arterial involvement, or (3) irreversible electroporation.

In the absence of metastatic disease, surgical exploration is dictated by the area of highest risk for an R1 resection. For example, if preoperative imaging shows soft tissue around the superior mesenteric artery, then this area needs to be addressed early in the operation, especially if an arterial divestment will be attempted. The ligament of Treitz is taken down and the length of the superior mesenteric artery is dissected out and sent for frozen section.

In borderline and locally advanced phase II trials, an R0 resection was achieved in $97 \%$ and $88 \%$ of patients, respectively, by sharply and bluntly dissecting the arterial adventitia and developing a plane. ${ }^{34}$ This tissue plane is developed on the artery proximal and distal to the cancer and is then followed under the cancer for the circumference and length of the involved artery. Since 2011, our institution has used this technique, which is similar to the arterial divestment technique published by $\mathrm{Yi}$ et al. ${ }^{35}$ Because the arterial wall is usually unaffected by microscopic tumor invasion, we feel that radical tumor clearance can be achieved without arterial resection. Multiple samples during this dissection are submitted for frozen section. In retrospective series of locally advanced PDAC, the 90 -day morbidity and mortality rates were $54.3 \%$ and $0.8 \%$, respectively. ${ }^{8,14,35}$ These results are in stark contrast to series that used arterial resection strategies that are associated with postoperative morbidity and mortality rates ranging from $36 \%$ to $52.2 \%$ and $2.9 \%$ to $>9 \%$, respectively. ${ }^{8,14,36}$

The most extensive experience of vascular resection and reconstruction is with superior mesenteric/portal vein involvement. If the cancer cannot be separated from a short venous segment, then resection with primary repair of the defect, vein patch, or segmental resection with end-to-end anastomosis should be considered. Longsegment venous involvement can be reconstructed with the interposition of an autologous or synthetic graft (Figure 2). ${ }^{37}$ Early on, pancreatectomy with venous resection and reconstruction was controversial, with opponents suggesting that it represented aggressive tumor biology and that venous resection and reconstruction may harm patients more than help them. However, more recent studies have shown that histopathologic evidence of venous invasion was not associated with worse survival when compared with results from patients without venous involvement, suggesting that venous invasion is more likely to be related to topography rather than biology. ${ }^{38,39}$ Moreover, as centers gathered more experience with the procedure, patients requiring venous resection and reconstruction had similar morbidity, mortality, and long-term survival when compared with those who did not require venous resection and reconstruction. ${ }^{40-43}$
A

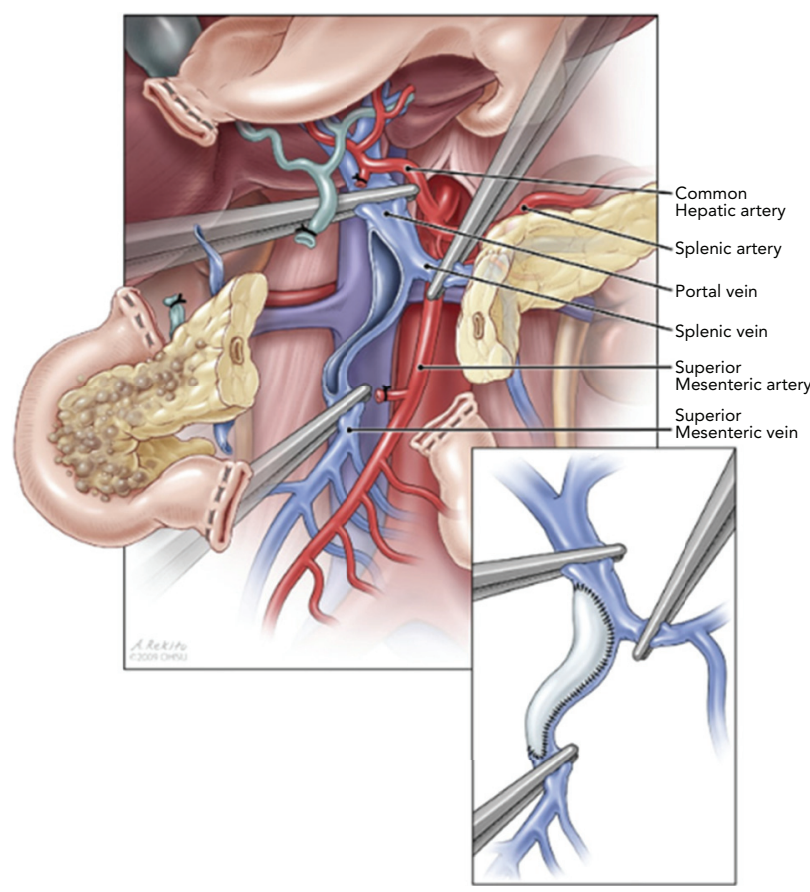

B

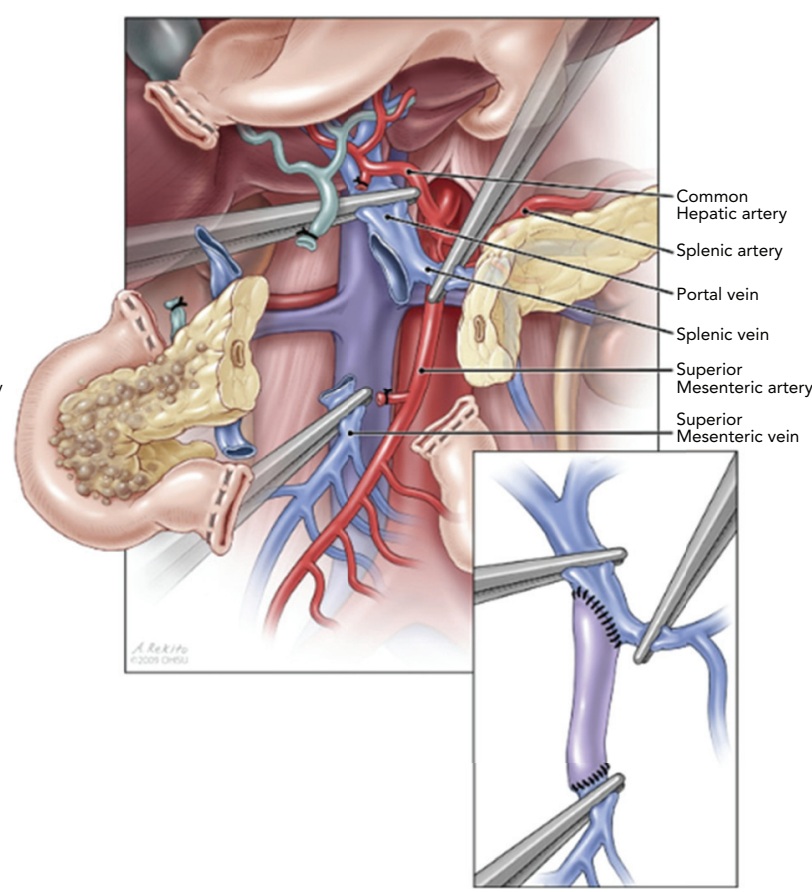

Figure 2. Portal/Superior mesenteric venous resection and reconstruction using (A) a vein patch and (B) a femoral vein interposition graft. From Lee DY, Mitchell EL, Jones MA, et al. Techniques and results of portal vein/superior mesenteric vein reconstruction using femoral and saphenous vein during pancreaticoduodenectomy. J Vasc Surg 2010;51:663; with permission. 
Management strategies surrounding tumors with arterial involvement are more complex and controversial. Certain groups have advocated for en bloc resection of the involved artery with reconstruction, reporting acceptable morbidity and mortality in a highly selected patient population. Depending on the artery involved, this can range from a modified Appleby procedure (distal pancreatectomy with celiac axis resection) for celiac artery involvement to a segmental resection with end-to-end anastomosis for superior mesenteric artery or common hepatic artery involvement (Figure 3).

Arterial resections without reconstruction, involving resection of the celiac trunk (Appleby procedure), are indicated in patients with pancreatic body cancers not involving the gastroduodenal artery who have experienced response to neoadjuvant therapy. ${ }^{44}$ Patients undergoing this operation after neoadjuvant therapy have shown significant improvement in 5-year OS (78.8\%) compared with those undergoing an upfront operation $(26.7 \%) .{ }^{45}$ Although this operation does not involve arterial reconstruction, it was associated with a high overall morbidity of $27 \%$ and a mortality of $9.5 \%$ in a multicenter retrospective analysis of 174 patients. ${ }^{46}$ Inadequate perfusion of the liver or the stomach after the resection are devastating complications from this operation. Truty et $\mathrm{al}^{47}$ recently reported outcomes of pancreatectomy with en bloc celiac axis resection among 90 patients with PDAC, of whom $89 \%$ had received neoadjuvant chemotherapy. The postoperative morbidity and mortality were

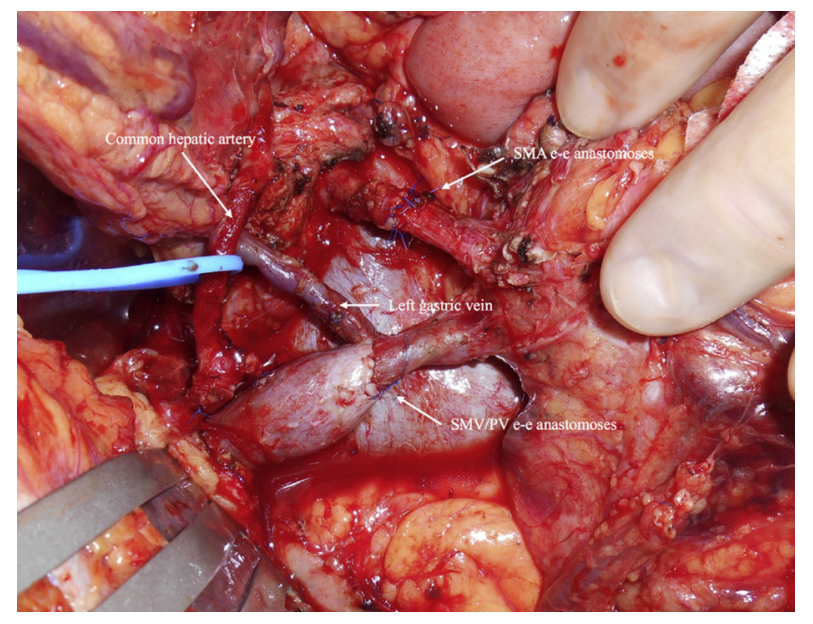

Figure 3. Total pancreatectomy with simultaneous superior mesenteric artery and superior mesenteric/portal vein resection and reconstruction in a patient with locally advanced PDAC who was preoperatively treated with FOLFIRINOX.

Abbreviations: e-e, end-to-end; FOLFIRINOX, fluorouracil/folinic acid/irinotecan/oxaliplatin; PDAC, pancreatic ductal adenocarcinoma; PV, portal vein; SMA, superior mesenteric artery; SMV, superior mesenteric vein.

From Del Chiaro M, Rangelova E, Halimi A, et al. Pancreatectomy with arterial resection is superior to palliation in patients with borderline resectable or locally advanced pancreatic cancer. HPB (Oxford) 2019;21:221; with permission.
$55 \%$ and $10 \%$, respectively, although the mortality rate for the last 50 patients was $4 \%$. Median recurrence-free survival and OS were 17.3 and 36.2 months, respectively.

Arterial resection with reconstruction continues to be actively explored in patients who have responded to neoadjuvant therapy. Unlike venous resection and reconstruction, arterial resection and reconstruction are associated with morbidity rates up to $54 \%$ and mortality rates ranging from $0 \%$ to $13 \% .{ }^{36,48}$ The actual improvement in OS for patients undergoing arterial resection is being explored, with 1 retrospective series documenting a median OS of 13.7 months for 118 patients undergoing arterial resection. ${ }^{49}$

\section{Positive Resection Margin Management Strategies}

When a negative margin cannot be achieved without arterial resection and reconstruction, we recommend an $\mathrm{R} 1$ resection with IORT if available. IORT is a valuable adjunct for local disease control. Patients deemed to have a higher likelihood of positive resection margins or an aborted resection due to extensive arterial involvement are surgically explored in a custom operating room with IORT capabilities (electron-beam linear accelerator). The goal of IORT is to improve local control by delivering high doses of radiation (10 Gy if resection performed, 15 Gy if inoperable) to the pancreatic bed while minimizing radiotoxicity by retracting and shielding normal surrounding tissue and organs. ${ }^{50,51}$

Furthermore, the addition of IORT in a cohort that has already received neoadjuvant chemotherapy followed by chemoradiation has not increased postoperative morbidity or mortality, but has produced encouraging local control rates in patients with borderline or locally advanced disease. For 158 patients with borderline or locally advanced PDAC who received IORT after neoadjuvant treatment, the median PFS and OS achieved were 21.5 and 46.7 months, respectively, if a surgical resection was performed. ${ }^{52}$ In patients found to be unresectable, IORT was associated with PFS and OS of 14.7 and 23 months, respectively. The overall major complication rates were $13 \%$ and $5 \%$ for IORT with surgical resection and for IORT alone, respectively. ${ }^{53}$

To summarize, IORT combined with surgical resection in patients with positive or close margins, or in those whose disease is unresectable due to locally advanced PDAC, has shown encouraging results. However, note that the aforementioned findings are largely our singleinstitution experience and need to be corroborated in future studies. The PACER trial is currently underway in collaboration with The Ohio State University, the Mayo Clinic, the University of North Carolina, and the University of California, Irvine, which will hopefully further our understanding of the utility of IORT. Figure 4 details our treatment algorithm in patients with locally advanced 
PDAC when assessing their appropriateness for surgical exploration and intraoperative decision-making.

Another modality that has been investigated in locally advanced PDAC is irreversible electroporation (IRE), a nonthermal ablation technique. The morbidity, mortality, and survival data have varied tremendously among groups. Although some groups have shown IRE to be safe $^{54}$ and to lead to improved survival over chemotherapy

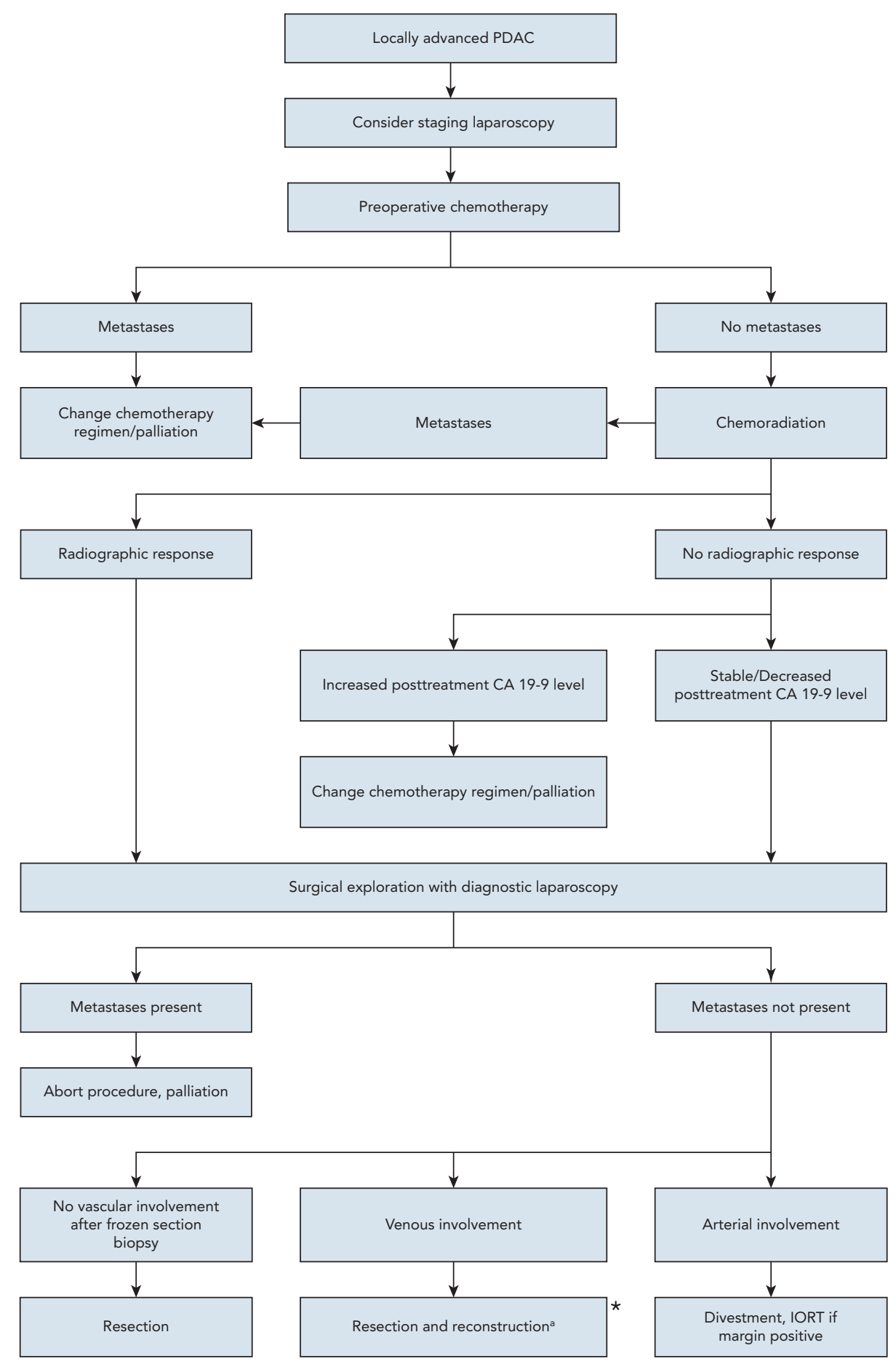

Figure 4. Treatment algorithm in patients with locally advanced PDAC when assessing their appropriateness for surgical exploration and considering intraoperative decision-making.

Abbreviations: IORT, intraoperative radiation therapy; PDAC, pancreatic ductal adenocarcinoma.

alf there is complete vein occlusion with cavernous transformation, then resection should be aborted. 
and radiation alone, ${ }^{55}$ others have reported significant morbidity (visceral ulcerations and perforations, portal vein thrombosis) and increased mortality rates with this procedure. ${ }^{56}$ The IMPALA prospective cohort study is a more contemporary analysis that assessed patients with locally advanced PDAC who underwent preoperative FOLFIRINOX therapy and showed that IRE offered no benefit over surgical resection but was associated with considerable morbidity (44\% and $11 \%$ major complication and mortality rates, respectively).$^{56}$ Our group feels that in the absence of randomized controlled trial data, IRE should be reserved for highly selected patients in the setting of a clinical trial at centers with experience with the technology.

\section{Conclusions}

The increased utilization of preoperative multiagent chemotherapy followed by chemoradiation therapy has significantly expanded the pool of patients with locally advanced PDAC who are eligible for resection with curative intent. Posttreatment cross-sectional imaging has been proven to not be a reliable indicator of resectability due to its inability to distinguish posttreatment fibrosis from residual viable tumor. Conversely, posttreatment CA 19-9 levels are predictive of tumor regression and should be used to guide decision-making regarding suitability for surgical exploration for resection. Diagnostic laparoscopy should be routinely used to minimize nontherapeutic laparotomy rates. For patients with arterial involvement, arterial divestment shows a lower morbidity and mortality rate than does arterial resection and reconstruction. In the setting of intraoperative unresectability or positive margins on frozen section analysis, IORT can be considered a safe and effective treatment option. The improvements in systemic therapy have significantly improved the resectability and OS of patients with locally advanced PDAC.

Submitted August 23, 2020; accepted for publication January 14, 2021.

Disclosures: The authors have not received any financial consideration from any person or organization to support the preparation, analysis, results, or discussion of this article.

Correspondence: Cristina R. Ferrone, MD, Department of Surgery, Massachusetts General Hospital, 15 Parkman Street, Boston, MA 02114-3117. Email: cferrone@mgh.harvard.edu

\section{References}

1. Siegel RL, Miller KD, Jemal A. Cancer statistics, 2020. CA Cancer J Clin 2020;70:7-30.

2. Rahib L, Smith BD, Aizenberg R, et al. Projecting cancer incidence and deaths to 2030: the unexpected burden of thyroid, liver, and pancreas cancers in the United States. Cancer Res 2014;74:2913-2921.

3. Arvold ND, Ryan DP, Niemierko A, et al. Long-term outcomes of neoadjuvant chemotherapy before chemoradiation for locally advanced pancreatic cancer. Cancer 2012;118:3026-3035.

4. Conroy $T$, Desseigne $F$, Ychou M, et al. FOLFIRINOX versus gemcitabine for metastatic pancreatic cancer. N Engl J Med 2011;364:1817-1825.

5. Von Hoff DD, Ervin T, Arena FP, et al. Increased survival in pancreatic cancer with nab-paclitaxel plus gemcitabine. N Engl J Med 2013;369: 1691-1703.

6. Sultana A, Smith CT, Cunningham D, et al. Meta-analyses of chemotherapy for locally advanced and metastatic pancreatic cancer. J Clin Oncol 2007;25:2607-2615

7. Gillen S, Schuster T, Meyer Zum Büschenfelde C, et al. Preoperative/neoadjuvant therapy in pancreatic cancer: a systematic review and meta-analysis of response and resection percentages. PLoS Med 2010;7: e1000267.

8. Hackert T, Sachsenmaier M, Hinz U, et al. Locally advanced pancreatic cancer: neoadjuvant therapy with FOLFIRINOX results in resectability in $60 \%$ of the patients. Ann Surg 2016;264:457-463.

9. Ferrone CR, Marchegiani G, Hong TS, et al. Radiological and surgical implications of neoadjuvant treatment with FOLFIRINOX for locally advanced and borderline resectable pancreatic cancer. Ann Surg 2015;261: 12-17.

10. Michelakos T, Pergolini I, Castillo CF, et al. Predictors of resectability and survival in patients with borderline and locally advanced pancreatic cancer who underwent neoadjuvant treatment with FOLFIRINOX. Ann Surg 2019;269:733-740.

11. Murphy JE, Wo JY, Ryan DP, et al. Total neoadjuvant therapy with FOLFIRINOX in combination with losartan followed by chemoradiotherapy for locally advanced pancreatic cancer: a phase 2 clinical trial. JAMA Oncol 2019;5:1020-1027.

12. Pawlik TM, Laheru D, Hruban $\mathrm{RH}$, et al. Evaluating the impact of a singleday multidisciplinary clinic on the management of pancreatic cancer. Ann Surg Oncol 2008;15:2081-2088.
13. Wagner $\mathrm{M}$, Antunes $\mathrm{C}$, Pietrasz $\mathrm{D}$, et al. CT evaluation after neoadjuvant FOLFIRINOX chemotherapy for borderline and locally advanced pancreatic adenocarcinoma. Eur Radiol 2017;27:3104-3116.

14. Truty MJ, Kendrick ML, Nagorney DM, et al. Factors predicting response, perioperative outcomes, and survival following total neoadjuvant therapy for borderline/locally advanced pancreatic cancer. Ann Surg $2021 ; 273: 341-349$

15. van Veldhuisen E, Vogel JA, Klompmaker S, et al. Added value of CA199 response in predicting resectability of locally advanced pancreatic cancer following induction chemotherapy. HPB (Oxford) 2018;20:605-611.

16. Xia BT, Fu B, Wang J, et al. Does radiologic response correlate to pathologic response in patients undergoing neoadjuvant therapy for borderline resectable pancreatic malignancy? J Surg Oncol 2017;115:376-383.

17. White RR, Paulson EK, Freed KS, et al. Staging of pancreatic cancer before and after neoadjuvant chemoradiation. J Gastrointest Surg 2001;5: 626-633.

18. Khawandanah MO, Kurkjian C, Penaroza S, et al. Utility of [18F] fluorodeoxyglucose positron emission tomography (FDG-PET) to predict resectability after neoadjuvant therapy in patients with unresectable pancreatic cancer on CT scans [abstract]. J Clin Oncol 2014;32(Suppl): Abstract 357

19. Garg I, Samreen N, Fletcher J, et al. Relationship between metabolic response on integrated time-of-flight FDG PET/MRI and pathologic treatment response in patients with FDG-avid borderline resectable pancreas cancer undergoing neoadjuvant therapy: a feasibility study [abstract]. J Nucl Med 2018;59(Suppl 1):Abstract 1406.

20. Wang ZJ, Behr S, Consunji MV, et al. Early response assessment in pancreatic ductal adenocarcinoma through integrated PET/MRI. Am J Roentgenol 2018;211:1010-1019.

21. Kaissis $G$, Ziegelmayer $S$, Lohöfer $F$, et al. A machine learning algorithm predicts molecular subtypes in pancreatic ductal adenocarcinoma with differential response to gemcitabine-based versus FOLFIRINOX chemotherapy. PLoS One 2019;14:e0218642.

22. Attiyeh MA, Chakraborty J, Mclntyre CA, et al. CT radiomics associations with genotype and stromal content in pancreatic ductal adenocarcinoma. Abdom Radiol (NY) 2019;44:3148-3157.

23. Boone BA, Steve J, Zenati MS, et al. Serum CA 19-9 response to neoadjuvant therapy is associated with outcome in pancreatic adenocarcinoma. Ann Surg Oncol 2014;21:4351-4358. 
24. Tsai S, George B, Wittmann D, et al. Importance of normalization of CA19-9 levels following neoadjuvant therapy in patients with localized pancreatic cancer. Ann Surg 2020;271:740-747.

25. Tempero MA, Malafa MP, Al-Hawary M, et al. NCCN Clinical Practice Guidelines in Oncology: Pancreatic Adenocarcinoma. Version 1.2020. Accessed January 18, 2021. To view the most recent version, visit NCCN.org

26. Tempero MA, Uchida E, Takasaki H, et al. Relationship of carbohydrate antigen 19-9 and Lewis antigens in pancreatic cancer. Cancer Res 1987; 47:5501-5503.

27. Fong ZV, Winter JM. Biomarkers in pancreatic cancer: diagnostic, prognostic, and predictive. Cancer J 2012;18:530-538.

28. Tjensvoll K, Lapin M, Buhl $\mathrm{T}$, et al. Clinical relevance of circulating KRAS mutated DNA in plasma from patients with advanced pancreatic cancer Mol Oncol 2016;10:635-643.

29. Perets $R$, Greenberg $O$, Shentzer $T$, et al. Mutant KRAS circulating tumo DNA is an accurate tool for pancreatic cancer monitoring. Oncologist 2018;23:566-572.

30. Cheng $\mathrm{H}$, Liu $\mathrm{C}$, Jiang $\mathrm{J}$, et al. Analysis of ctDNA to predict prognosis and monitor treatment responses in metastatic pancreatic cancer patients. Int J Cancer 2017;140:2344-2350.

31. Fong ZV, Alvino DML, Fernández-Del Castillo C, et al. Reappraisal of staging laparoscopy for patients with pancreatic adenocarcinoma: a contemporary analysis of 1001 patients. Ann Surg Oncol 2017;24: 3203-3211.

32. Sell NM, Fong ZV, Del Castillo CF, et al. Staging laparoscopy not only saves patients an incision, but may also help them live longer. Ann Surg Oncol 2018:25:1009-1016.

33. Peng JS, Mino J, Monteiro R, et al. Diagnostic laparoscopy prior to neoadjuvant therapy in pancreatic cancer is high yield: an analysis of outcomes and costs. J Gastrointest Surg 2017;21:1420-1427.

34. Zhang B, Lee GC, Qadan M, et al. Revision of pancreatic neck margins based on intraoperative frozen section analysis is associated with improved survival in patients undergoing pancreatectomy for ductal adenocarcinoma [published online December 5, 2019]. Ann Surg, doi: 10.1097/SLA.0000000000003503

35. Yi M, Kuirong J, Baobao C, et al. Artery divestment for artery involved pancreatic cancer: a retrospective study. Pancreatology 2017;17(Suppl 4): S25-26.

36. Del Chiaro M, Rangelova E, Halimi A, et al. Pancreatectomy with arterial resection is superior to palliation in patients with borderline resectable or locally advanced pancreatic cancer. HPB (Oxford) 2019;21:219-225.

37. Lee DY, Mitchell EL, Jones MA, et al. Techniques and results of portal vein/superior mesenteric vein reconstruction using femoral and saphenous vein during pancreaticoduodenectomy. J Vasc Surg 2010;51: 662-666.

38. Tseng JF, Raut CP, Lee JE, et al. Pancreaticoduodenectomy with vascular resection: margin status and survival duration. J Gastrointest Surg 2004; 8:935-950.

39. Yekebas EF, Bogoevski D, Cataldegirmen G, et al. En bloc vascular resection for locally advanced pancreatic malignancies infiltrating major blood vessels: perioperative outcome and long-term survival in 136 patients. Ann Surg 2008;247:300-309.

40. Ravikumar R, Sabin C, Abu Hilal M, et al. Portal vein resection in borderline resectable pancreatic cancer: a United Kingdom multicenter study. J Am Coll Surg 2014;218:401-411.

41. Zhou $Y$, Zhang Z, Liu $Y$, et al. Pancreatectomy combined with superior mesenteric vein-portal vein resection for pancreatic cancer: a metaanalysis. World J Surg 2012;36:884-891.
42. Yu SL, Zhang GF, Ye C et al. Survival determinants after pancreatectomy with vascular resection for pancreatic cancer. Int Surg 2016;101:443-452.

43. Tsiotos GG, Ballian N, Michelakos T, et al. Portal-mesenteric vein resection in borderline pancreatic cancer; 33 month-survival in patients with good performance status. J Pancreat Cancer 2019;5:43-50.

44. Peters NA, Javed AA, Cameron JL, et al. Modified Appleby procedure for pancreatic adenocarcinoma: does improved neoadjuvant therapy warrant such an aggressive approach? Ann Surg Oncol 2016;23:3757-3764.

45. Nakamura T, Hirano S, Noji T, et al. Distal pancreatectomy with en bloc celiac axis resection (modified Appleby procedure) for locally advanced pancreatic body cancer: a single-center review of 80 consecutive patients. Ann Surg Oncol 2016;23(Suppl 5):969-975.

46. Klompmaker S, Peters NA, van Hilst J, et al. Outcomes and risk score for distal pancreatectomy with celiac axis resection (DP-CAR): an international multicenter analysis. Ann Surg Oncol 2019;26:772-781.

47. Truty MJ, Colglazier JJ, Mendes BC, et al. En bloc celiac axis resection for pancreatic cancer: classification of anatomical variants based on tumor extent. J Am Coll Surg 2020;231:8-29.

48. Tee MC, Krajewski AC, Groeschl RT, et al. Indications and perioperative outcomes for pancreatectomy with arterial resection. J Am Coll Surg 2018;227:255-269.

49. Bachellier $P$, Addeo $P$, Faitot $F$, et al. Pancreatectomy with arterial resection for pancreatic adenocarcinoma: how can it be done safely and with which outcomes? A single institution's experience with 118 patients. Ann Surg 2020;271:932-940.

50. Cai S, Hong TS, Goldberg SI, et al. Updated long-term outcomes and prognostic factors for patients with unresectable locally advanced pancreatic cancer treated with intraoperative radiotherapy at the Massachusetts General Hospital, 1978 to 2010. Cancer 2013;119:4196-4204.

51. Chen $Y$, Che $X$, Zhang J, et al. Long-term results of intraoperative electron beam radiation therapy for nonmetastatic locally advanced pancreatic cancer: retrospective cohort study, 7-year experience with 247 patients at the National Cancer Center in China. Medicine (Baltimore) 2016;95: e4861.

52. Harrison JM, Wo JY, Ferrone CR, et al. Intraoperative radiation therapy (IORT) for borderline resectable and locally advanced pancreatic ductal adenocarcinoma (BR/LA PDAC) in the era of modern neoadjuvant treatment: short-term and long-term outcomes. Ann Surg Oncol 2020;27: 1400-1406.

53. Martin RCG II, McFarland K, Ellis S, et al. Irreversible electroporation therapy in the management of locally advanced pancreatic adenocarcinoma. J Am Coll Surg 2012;215:361-369.

54. Martin RCG II, McFarland K, Ellis S, et al. Irreversible electroporation in locally advanced pancreatic cancer: potential improved overall survival. Ann Surg Oncol 2013;20(Suppl 3):S443-449.

55. Kluger MD, Epelboym I, Schrope BA, et al. Single-institution experience with irreversible electroporation for T4 pancreatic cancer: first 50 patients. Ann Surg Oncol 2016;23:1736-1743.

56. Vogel JA, Rombouts SJ, de Rooij T, et al. Induction chemotherapy followed by resection or irreversible electroporation in locally advanced pancreatic cancer (IMPALA): a prospective cohort study. Ann Surg Oncol 2017;24:2734-2743.

57. Callery MP, Chang KJ, Fishman EK, et al. Pretreatment assessment of resectable and borderline resectable pancreatic cancer: expert consensus statement. Ann Surg Oncol 2009;16:1727-1733.

58. Katz MHG, Marsh R, Herman JM, et al. Borderline resectable pancreatic cancer: need for standardization and methods for optimal clinical tria design. Ann Surg Oncol 2013;20:2787-2795. 